\title{
MODELLING THE INFLUENCE OF INLET ANGLE CHANGE ON THE PERFORMANCE OF SUBMERSIBLE WELL PUMPS
}

\author{
Wang, H. L. ; Long, B. ; Yang, Y.** Xiao, Y. ${ }^{* *} \&$ Wang, C. ${ }^{* * *, \#}$ \\ *School of Aerospace and Mechanical Engineering/Flight College, Changzhou Institute of \\ Technology, Changzhou, 213032, China \\ ${ }^{* *}$ National Research Center of Pumps, Jiangsu University, Zhenjiang, 212013, China \\ *** College of Hydraulic Science and Engineering, Yangzhou University, Yangzhou, 225009, China \\ E-Mail: wangchuan@ujs.edu.cn $\left({ }^{\#}\right.$ Corresponding author $)$
}

\begin{abstract}
Three groups of schemes were designed for different blade inlet angles of impellers to improve the efficiency and single-stage head of submersible well pumps. Furthermore, the hydraulic performance of the pumps was analysed using ANSYS CFX. As a result, the heads and efficiencies of the schemes were acquired. The internal flow fields of the schemes under different flow quantities were analysed, and the accuracy of the optimal scheme was experimentally verified. Results indicated that enlarging the inlet angle of the blade of submersible well pumps might improve the hydraulic performance to a certain degree. In comparison with the general design scheme with a fixed inlet angle that with an inlet angle was $26^{\circ}$ at the front cover plate and $20^{\circ}$ at the rear cover plate, and that in the middle was under uniform change. The incoming flow angle of the medium coincided with the inlet angle; thus, the discharge capacity of the pump had a high matching ratio with the actual discharge capacity. The head and efficiency improved remarkably relative to the initial model, and thus, the hydraulic performance of the pump improved.

(Received in November 2019, accepted in March 2020. This paper was with the authors 2 months for 1 revision.)
\end{abstract}

Key Words: $\quad$ Submersible Well Pumps, Inlet Angle, Hydraulic Design, Internal Flow Field

\section{INTRODUCTION}

As the core equipment used to extract underground water, submersible well pumps have broad application prospect in various fields, such as agricultural irrigation, sewage treatment, and oilfield exploitation [1-3]. Hydraulic performance [4-6] is an important index for evaluating pump performance, and the performance loss caused by the inappropriate selection of many geometric parameters considerably affect hydraulic performance [7-8]. Bacharoudis et al. [9] analysed the performance of impellers with different blade outlet angles under the same outlet diameter. Then, these scholars calculated the flow conditions in the blade channel, analysed and summarized the prediction curve and calculation results of the internal flow field, and selected the optimal parameters to improve the hydraulic performance. Currently, submersible well pumps are usually designed via numerical simulation [10-12], the hydraulic loss method, and an approximation model.

On this basis, research scholars have explored the influence of the design parameters of submersible well pumps on hydraulic performance [13-15]. However, few in-depth studies regarding the hydraulic performance of submersible well pumps have been performed based on the design of the inlet angle of the impeller. Therefore, the influence of the inlet angle design of submersible well pumps and the change in flow field must be investigated. Design schemes with different inlet angles are selected, and CFD software was used to perform the numerical calculation and investigate the external characteristics of internal flow field in these schemes. The hydraulic performance of the schemes is then compared to guide submersible well pump designers with a theoretical design. 


\section{STATE OF THE ART}

Many experts and scholars worldwide have conducted substantial research on the influence of the design parameters of centrifugal pumps on hydraulic design. Stel et al. [16] used the numerical simulation method to investigate a multistage submersible pump and discovered that the accurate hydraulic performance of pumps above three stages could be acquired through the numerical simulation of a three-stage pump. On this basis, the present study conducted a numerical simulation analysis and research on submersible well pumps and investigated the hydraulic performance of three-stage submersible well pumps. El-Gazzar and Hawash [17] studied the design method of improving hydraulic performance through the impeller blade splitting method of a centrifugal pump; however, the eccentricity problem caused by this method influenced the rotation centre of the pump to some degree, resulting in a great vibration amplitude and increase in radial force. Only blade placement was considered in the present study, but the structural changes caused by the change of each segment of the splitter blade angle were not associated. Gölcü et al. [18] studied the influence of a splitter blade in a deep-well pump on hydraulic performance. Then, these scholars conducted a comparative analysis of the influences of the changes in quantity and length of the splitter blades on the performance through numerical simulation. The results revealed that splitter blades of a certain quantity and $80 \%$ blade length could effectively elevate the head, but excessive splitter blades would block the flow channel and reduce pump efficiency. The overall placement of splitter blades was investigated without an intensive analysis of whether the placement angles of the original and splitter blades could weaken the blocking problem to a certain degree while elevating the head. Wei and Sun [19] studied the influence of the diffuser inlet's width on the performance of submersible pumps and found that increases of the inlet's width could effectively offset the large energy, reduce the hydraulic loss of the diffuser, and improve the hydraulic efficiency of the entire pump as the inlet width increases. However, multistage impellers in submersible well pumps are interconnected by diffusers; thus, the flow field distribution at the impeller inlet since the second stage is determined by the diffuser design quality, whereas quality influences the hydraulic performance of the multistage submersible pump. Ajay et al. [20] optimized the impeller shape based on many optimization algorithms and predicted the optimization result by comparing the head losses, thereby reducing experimental time to a certain degree. Based on this method, a follow-up research was conducted in simultaneous consideration of the blade inlet and outlet angles. Lu et al. [21] conducted an experimental study that combined the orthogonal, grey correlation, and selfpriming time tests to investigate the influencing factors of the self-priming time of a multistage self-priming centrifugal pump and shorten the corresponding self-priming time. However, the influence of impeller blade inlet parameters on self-priming performance was not analysed. In order to improve the hydrodynamic performance of the centrifugal pump, Zhou et al. [22] made an orthogonal experiment to optimize the impeller design parameters. Five main impeller geometric parameters were chosen as the research factors, and 16 impellers were designed. Through the variance analysis method, the best parameter combination for higher efficiency was captured finally. However, the 16 impellers equipped with the same volute. The matching of impeller and guide vane was not studied. Kim et al. [23] implemented an optimization design of the inlet and outlet angles of the blades of a centrifugal pump impeller based on the response surface method, thereby effectively improving hydraulic efficiency. However, optimization was only achieved by setting fixed values of the inlet and outlet angles at the intersecting lines of the blade's front and rear cover plates; the influence of the possible linear change from the intersecting line of the front cover plate to that of the rear cover plate on the hydraulic performance of the pump was not considered. Li et al. [24] studied the rotor-stator interaction mechanism in impeller and guide vanes of mixed-flow pump under part loading condition, the flow field between the impeller 
outlet and the guide vane inlet under part loading condition was measured based on Particle Image Velocimetry (PIV). The research results provide significant reference value for revealing the internal flow characteristics under part loading condition as well as for optimization of mixed-flow pump. Influence of rotor-stator interaction on the flow in the area between the impeller outlet and the guide vane inlet was analysed according to the combination of the velocity and the vorticity distribution in the related area. However, the characteristic hydrodynamic structure of the rotor-stator interaction area was not suitable for submersible well pumps. Capurso et al. [25] proposed a novel impeller designed for lowmedium specific speed double suction centrifugal pumps, and found that the novel double suction impeller was characterized by a new arrangement of its flow channels, which come up alternately on the same circumferential exit even if they started from the two different sides. However, higher hydraulic blade loading should be designed to explore performance and the efficiency of the new geometry and to correlate the number of blades with the slip factor value for the novel design. Jiang et al. [26] analysed the influence of the axial dimensional error of the impeller and the guide vane on the external performance and internal flow of the pump. The results revealed that when the diffuser inlet width is less than the impeller outlet width, an increase in the diffuser inlet width always causes a reduction in the pump head, and the pump efficiency increases at a small flow rate but decreases at a large flow rate. However, the matching design method of impeller and diffuser was needed more experimental verification. Namazizadeh et al. [27] optimized the impeller of a centrifugal pump by investigating the effect of adding splitter blades and modifying their geometry. Using Design of Experiment (DoE) technique, the design space was created and response surface method was utilized to find the optimum geometry. The results showed adding splitters could improve total head by about $10.6 \%$ and by modifying the geometry using DoE technique it could increase further by $4.4 \%$ with the negligible effect on the pump overall efficiency. However, more different parameters should be considered and using Box-Behnken technique in DoE. More research was needed in the applicability of second order polynomial curves.

The above research results were mainly based on the numerical simulation prediction and optimization design of the internal structure of centrifugal pumps. However, most research on the inlet and outlet angles of impeller blades were under the uniform change situation. Research on the influence of inlet angle under a linear change on the hydraulic performance of submersible well pumps, although few, has been conducted. The optimization of the impeller blade is crucial to the control of the inlet flow field loss of the impeller and the hydraulic performance of the impeller channel. Therefore, a fluid computational domain model was established via numerical simulation. Starting from the influences of pump impeller parameters on the overall pump performance, this study explored the influence characteristics of different designed blade inlet angles on impeller performance and even the overall performance of submersible well pumps.

The remainder of this study is arranged as follows: Section 3 discusses the modelling for pump parameter optimization and mesh generation and the hydraulic performance analysis under multiple working conditions. Section 4 obtains the optimal scheme and the mechanism of parameter optimization through a comparative analysis of the flow fields and test data of different schemes. Section 5 summarizes the study and provides the conclusions.

\section{METHODOLOGY}

\subsection{Geometric model}

A 200QJ50 submersible well pump was taken as the study object, and a three-stage pump was taken as the calculation model. The hydraulic models of the impeller and the diffuser at each stage were the same. Their design parameters were as follows: rotation speed $n=2850 \mathrm{r} / \mathrm{min}$, 
design flow quantity $Q_{\text {des }}=50 \mathrm{~m}^{3} / \mathrm{h}$, single-stage head $H=17 \mathrm{~m}$, specific speed $n_{\mathrm{s}}=146.43$ (calculated in accordance with a single-stage head), outlet diameter $D_{2}=134 \mathrm{~mm}$, outlet width $b_{2}=16 \mathrm{~mm}$, number of impeller blades $Z=6$, and outlet angle $\beta_{2}=20.5^{\circ}$. A three-dimensional model of the impeller and the diffuser is shown in Fig. 1.
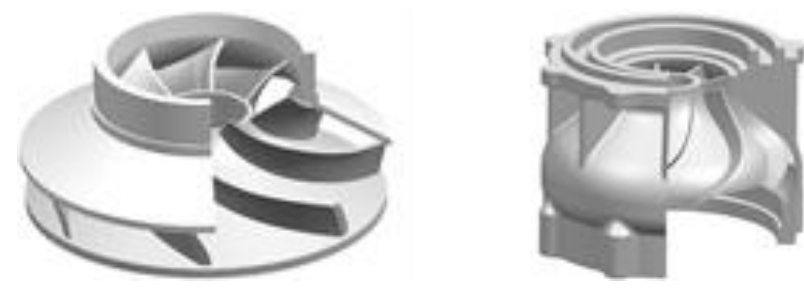

Figure 1: Three-dimensional model of the impeller and the diffuser.

Three different impeller inlet angle schemes were selected. The performance change of this submersible well pump under the three schemes and the distribution difference of the internal flow field were analysed through numerical calculation. The results provide a certain theoretical reference for the inverse design methods of submersible well pumps.

Among the three inlet angle schemes, the impeller inlet angle under the first scheme presented a continuously decreasing trend from the front cover plate to the rear cover plate; the inlet angle was $26^{\circ}$ at the front cover plate and $20^{\circ}$ at the rear cover plate, and that in the middle was under uniform change. The inlet angle under the second scheme was constant at $26^{\circ}$, and that under the third scheme was $20^{\circ}$. Table I shows the abovementioned inlet angle design schemes.

Table I: Design scheme of the inlet blade angle.

\begin{tabular}{|c|c|c|}
\hline \multirow{2}{*}{ Scheme } & \multicolumn{2}{|c|}{ Inlet angle $\boldsymbol{\beta}_{\mathbf{1}}$} \\
\cline { 2 - 3 } & Front cover & Cover plate \\
\hline $\mathbf{1}$ & $26^{\circ}$ & $20^{\circ}$ \\
\hline $\mathbf{2}$ & $26^{\circ}$ & $26^{\circ}$ \\
\hline $\mathbf{3}$ & $20^{\circ}$ & $20^{\circ}$ \\
\hline
\end{tabular}

\subsection{Numerical simulation}

ANSYS ICEM was used to generate high-quality hexahedral meshes in the computational domains of submersible well pumps because of the considerable effect of mesh quality on the numerical calculation. The meshes were added to each boundary while guaranteeing the quality of each part. Most submersible well pumps are multistage pumps with numerous meshes. Mesh independence analysis must be performed to guarantee calculation accuracy and improve calculation efficiency. Scheme 1 was selected for mesh generation and independence analysis. Fig. 2 shows a schematic of the computational meshes. The left side shows the single-channel meshes of the impeller, whereas the right side shows the single-channel meshes of the diffuser.

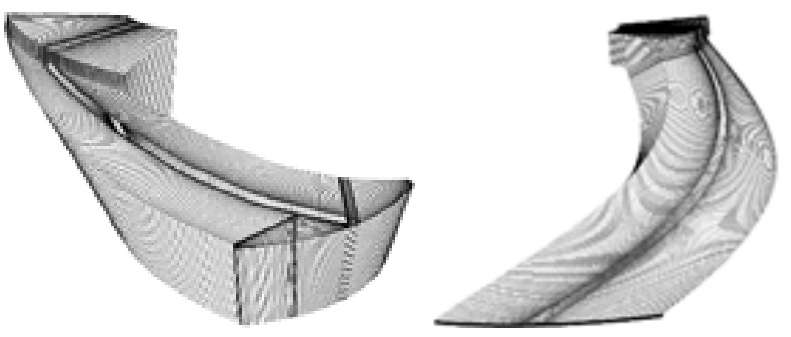

Figure 2: Mesh of the single channels.

Table II shows the independence analysis results of the meshes. When the global meshes were $1.6 \mathrm{~mm}$ in size, the simulation performance was approximate to the predicted 
performance. In consideration of calculation time and accuracy, the maximum global size in Scheme $\mathrm{C}$ was selected for mesh generation.

Table II: Analysis of grid independence.

\begin{tabular}{|c|c|c|c|}
\hline Scheme & Maximum grid size $(\mathrm{mm})$ & Grid number & Efficiency $(\%)$ \\
\hline A & 2.4 & $4.4812 \times 10^{6}$ & 72.12 \\
\hline B & 2.0 & $5.6395 \times 10^{6}$ & 73.88 \\
\hline C & 1.6 & $6.6512 \times 10^{6}$ & 75.09 \\
\hline D & 1.2 & $8.9851 \times 10^{6}$ & 75.12 \\
\hline
\end{tabular}

The steady-stage computation of multiple working conditions was performed for different impeller inlet angles via ANSYS-CFX 18.0 software. The total pressure inlet and the mass flow outlet were used for the computational domain. The impeller of this submersible well pump was a rotational domain, whereas that of the other parts such as diffuser in pumps were static. The standard $k-\varepsilon$ turbulence model was used, and the convergence precision of the residual error was set to $10^{-4}$. Nonslip boundary conditions were used for the wall surfaces, and the standard wall surface function was set. The computational subdomains were connected by setting the interfacial surfaces. The head, efficiency, and shaft power were monitored to guarantee the reliability of the calculation result.

The standard $k-\varepsilon$ model is a typical two-equation model that has been extensively applied in the simulation of flow field and heat exchange, and its equations can be expressed as follows:

$$
\begin{gathered}
\frac{\partial(\rho k)}{\partial t}+\frac{\partial\left(\rho k u_{i}\right)}{\partial t}=\frac{\partial}{\partial x_{j}}\left[\left(\mu+\frac{\mu_{i}}{\sigma_{k}}\right) \frac{\partial k}{\partial x_{j}}\right]+G_{k}+G_{b}-Y_{M}-\rho \varepsilon+S_{k} \\
\frac{\partial(\rho \varepsilon)}{\partial t}+\frac{\partial\left(\rho \varepsilon u_{i}\right)}{\partial x_{i}}=\frac{\partial}{\partial x_{j}}\left[\left(\mu+\frac{\mu_{i}}{\sigma_{\varepsilon}}\right) \frac{\partial \varepsilon}{\partial x_{j}}\right]+\frac{C_{1 \varepsilon} \varepsilon}{k}\left(G_{k}+G_{3 \varepsilon} G_{b}\right)-\rho C_{2 \varepsilon} \frac{\varepsilon^{2}}{k}+S_{\varepsilon}
\end{gathered}
$$

\subsection{Numerical simulation result}

Figs. 3 and 4 show the numerical simulation results of 200QJ50 submersible well pump. The comparison of the heads of the pump under the three schemes and the rated flow quantity revealed that the head of the submersible pump model in Scheme 1 was the highest, followed by those in Schemes 2 and 3 in succession; however, the head difference at the rated point was not evident. As the flow rate decreased, the increase in the amplitude of the head in Scheme 3 was larger than those in the other two schemes. Consequently, under the low flow rate $\left(0.6 Q_{\mathrm{des}}\right)$, the head in Scheme 3 exceeded those of the other two schemes, and then the model in this group had the highest hydraulic efficiency under a low flow rate. However, under a large flow rate (1.4 $\left.Q_{\text {des }}\right)$, the head of Scheme 3 was lower than those in Schemes 1 and 2. The high head under the low flow rate and the low head under large flow rate resulted in a steep head curve in Scheme 3 , and the overall scope of this working condition was smaller than those of the other two models. Therefore, the coverage of the flow rate of the efficiency curve in Scheme 3 was remarkably smaller than those of the other two schemes. Under the rated flow rate, the efficiency of Scheme 3 reached the maximum value. As the flow rate further increased, the efficiency curve of this model under a large flow rate declined to a great degree. In comparison with Scheme 3, the highest efficiency points of the submersible well pumps in Schemes 1 and 2 both existed under the $1.2 Q_{\text {des }}$ flow rate; thus, the coverage of the operating condition of this submersible well pump enlarged by a huge margin, and its hydraulic efficiency under a large flow rate improved. Therefore, in the design process of submersible well pumps, the inlet angle of the impeller should be appropriately enlarged under the $Q_{\text {des }}$ flow rate to contribute to a large scope of high-efficiency use condition of this submersible pump model. Furthermore, 
the increase in inlet angle would result in a clear shift in the extreme point on the efficiency curve of the submersible well pump toward the operating condition with a large flow rate. Such effect would considerably improve the efficiency of submersible well pumps under a large flow rate and slightly improve the efficiency under a low flow rate.

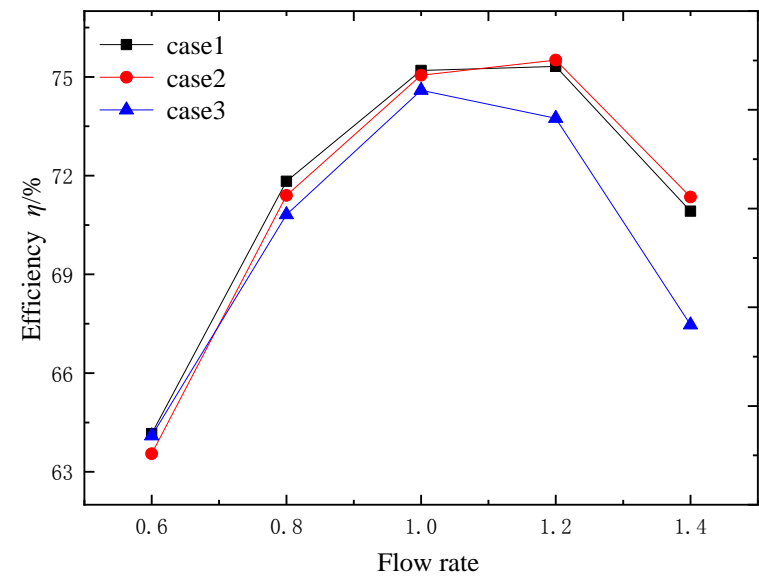

Figure 3: Prediction results of submersible well pump head for 200QJ50.

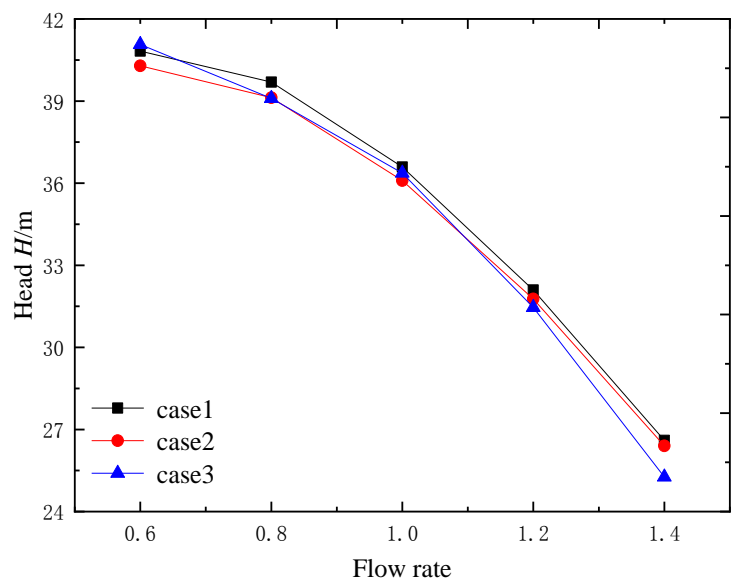

Figure 4: Prediction results of submersible well pump efficiency for 200QJ50.

The comparison of the performance of Scheme 1 with that of Scheme 2 shows that the head of Scheme 1 was slightly higher than that of Scheme 2 within the full scope of the flow rate. Moreover, the efficiency of Scheme 1 under the low flow rate was slightly higher than that of Scheme 2 under the rated flow rate due to the head difference. However, under a large flow rate, if a single value was taken for the inlet angle on the basis of the calculation result, the performance under large flow rate would certainly improve. However, the head and efficiency under the low flow rate could not satisfy the related requirements, and the axial power curve of the model would shift toward the large flow rate, thus weakening the nonoverload performance of the multistage pump.

\section{RESULT ANALYSIS AND DISCUSSION}

\subsection{Flow field analysis in pump}

The internal flow field distribution of the three groups of submersible well pump models under different flow conditions was further analysed to probe into the influence mechanism of the inlet angle on the performance of the submersible well pump and obtain the internal medium distribution in the submersible pump under different inlet angles.

Fig. 5 shows the unfolded streamline distribution graph of the cross section in the impeller flow channel of the three groups of submersible well pumps under the $0.6 Q_{\text {des }}$ flow rate. The actual rate of the impeller channel was far smaller than its discharge capacity. Thus, a flow separation phenomenon occurred near the blade back in the middle segment of the first-stage impeller channel in the three groups of models. Under the actions of flow separation and pressure difference, the medium flow inside the impeller channel was no longer a uniform laminated flow, and the secondary flow of the liquid medium perpendicular to the main flow direction appeared. The secondary flow was more evident in the impeller channel under Schemes 2 and 3 compared with that in Scheme 1. In addition, the secondary flow in the impeller channel caused a vortex in the latter half segment of the individual channel. This vortex might result in a large hydraulic loss. At the secondary impeller inlet of the three groups of models, the liquid medium maintained a certain peripheral speed after passing through the guide blade of the previous stage; thus, its incoming flow angle was larger than the inlet angle of the primary-stage impeller. Given the minimum inlet angle in Scheme 3, a mismatching 
phenomenon occurred between the medium's incoming flow angle and its blade inlet angle at the inlet of the secondary impeller; that is, the fluid tended to bypass the blade inlet rim due to the small inlet angle and then entered the side near the back face. Therefore, the secondary flow in the impeller channel occurred in advance, and a low-voltage vortex region was induced in the middle segment of the impeller channel. In Schemes 1 and 2, the difference between the incoming flow angle at the inlet and that at the primary stage also induced the occurrence of secondary flow in the channel in advance. The strength of the secondary flow was weaker than that of Scheme 3 because the inlet angle was large. A vortex was not induced by the secondary flow in Scheme 1. Even though a low-pressure vortex was induced in the flow channel of Scheme 2, the intensity of the vortex core was smaller than that in Scheme 3.

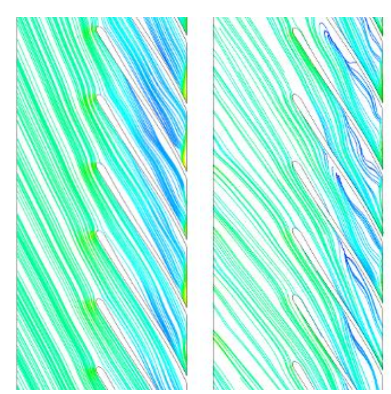

Scheme 1

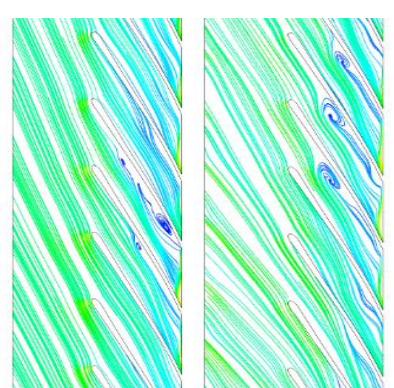

Scheme 2

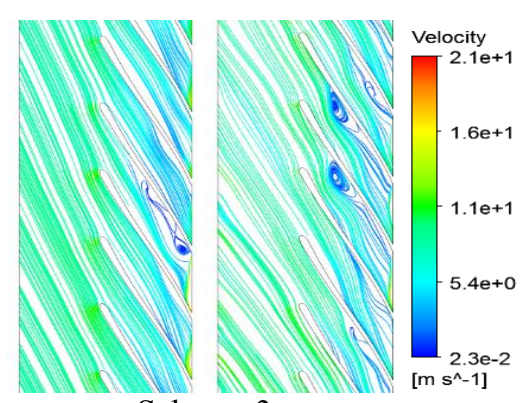

Scheme 3

Figure 5: Streamline distribution on the cross section of the impeller channel under $0.6 Q_{\text {des. }}$.

Figs. 6 and 7 show the unfolded streamline distribution graphs of the cross section in the impeller channel of the three groups of submersible well pump models under the $Q_{\text {des }}$ and 1.4 $Q_{\text {des }}$ flow rates. Under the $Q_{\text {des }}$ flow rate, the liquid medium distribution in the impeller channel improved greatly in comparison with that under a low flow rate. Moreover, its discharge capacity had a high matching ratio with the actual discharge rate. Therefore, even though the secondary flow caused by blade extrusion remained in the channel, the overall flow field distribution had evident layers, which could increase the hydraulic efficiency.

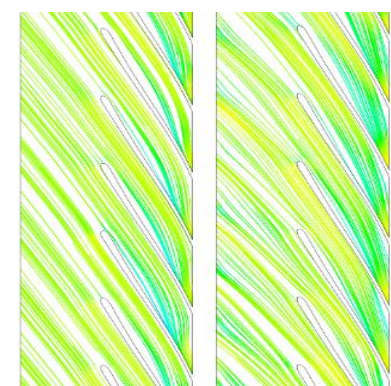

Scheme 1

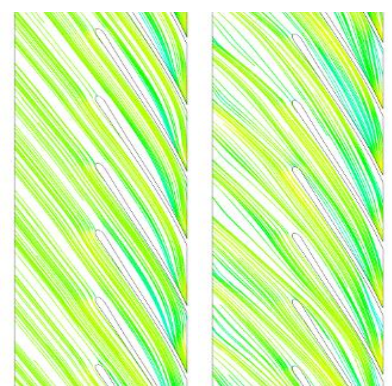

Scheme 2

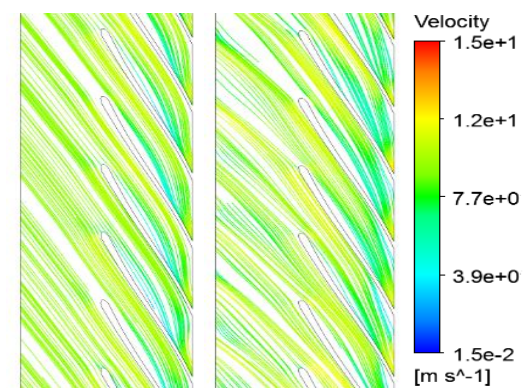

Scheme 3

Figure 6: Streamline distribution on the cross section of the impeller channel under $Q_{\text {des }}$.

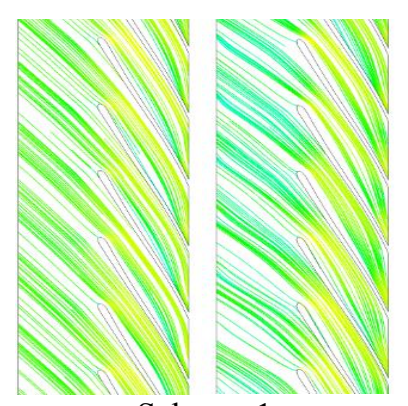

Scheme 1

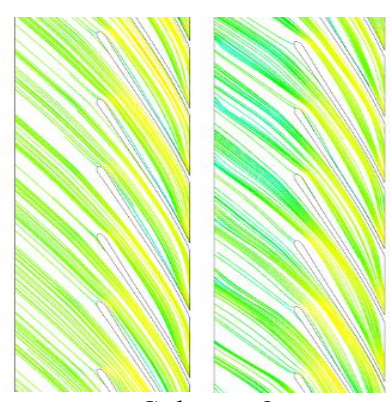

Scheme 2

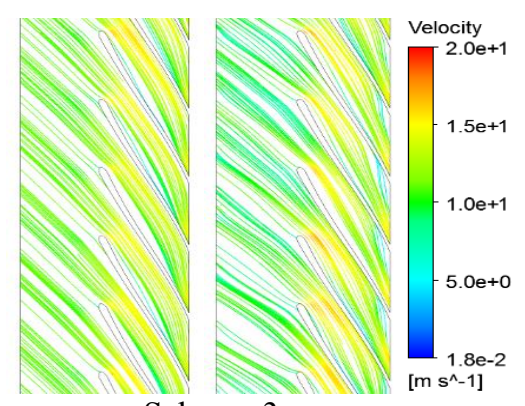

Scheme 3

Figure 7: Streamline distribution on the cross section of the impeller channel under1.4 $Q_{\text {des. }}$. 
As the flow rate increased, the actual discharge rate of the impeller exceeded the optimal discharge rate under the $1.4 Q_{\text {des }}$ flow rate. Hence, the extruding effect of the blade on the inlet's incoming flow was clear, and a high flow-rate region was formed near the working face in the first half segment of the impeller channel. Thus, the pressure on the working face of the impeller decreased greatly, inducing flow separation at the middle segment of the impeller. In the three groups of schemes, the inlet angle of Scheme 3 was the minimum, and the unmatching ratio between its blade and incoming flow angles was also the highest. Thus, the flow separation phenomenon in the last half segment of the channel was the most evident.

Figs. 8-10 show the static pressure distribution on the primary impeller blade of the three different inlet angle schemes under different flow quantities. Under the $0.6 Q_{\text {des }}$ flow rate, a low-pressure region was generated at the back face near the inlet rim of the impeller blade of the submersible well pump. This region was caused by the non-uniform local speed distribution of the liquid medium. The speed of the liquid medium at the inlet rim near the back face was higher than that at the side of the working face because of the extruding effect of the blade inlet rim. The static pressure distribution at this position was lower than at the other positions in the channel. Near the low-pressure core region near the inlet at the rim, the liquid medium could easily generate flow turbulence to diffuse inside the impeller channel. Such turbulence caused a secondary flow in the channel and induced a vortex. In the three groups of schemes, the coverage area of the low-pressure core region was smaller than those of other two schemes. The low-pressure region was mainly distributed at the lower half part at the inlet rim near the rear cover plate, and its minimum pressure was higher than those of the other schemes. In Schemes 2 and 3 , the pressure gradient distribution was large in the low-pressure region, and the coverage area was already extended to the blade inlet rim near the first half part of the front cover plate. Meanwhile, the diffusion trend toward the impeller channel was also manifested in the lowpressure region in Scheme 2. This result indicated that among the three groups of schemes, the parameter of the inlet angle used in scheme 1 has a better effect on reducing the low-pressure core region at the back face of the inlet rim. By contrast, selecting a large constant inlet angle (Scheme 2) resulted in the diffusion phenomenon of the low-pressure region inside the channel.

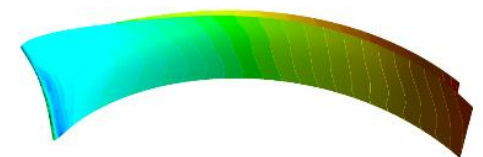

Scheme 1

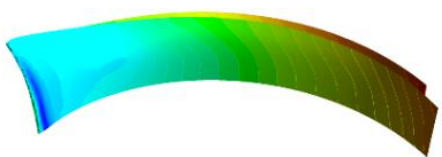

Scheme 2

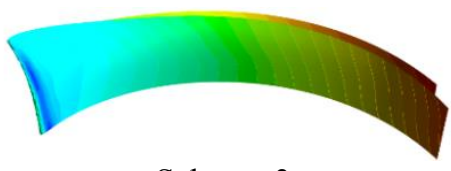

Scheme 3

Figure 8: Static pressure distribution on the blade of the first impeller under $0.6 Q_{\text {des. }}$.

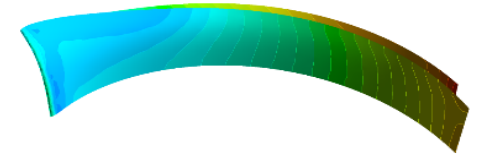

Scheme 1

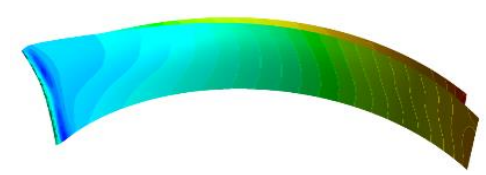

Scheme 2

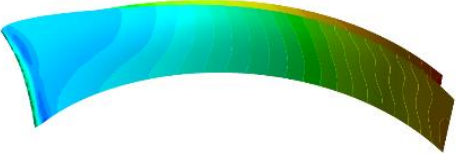

Scheme 3

Figure 9: Static pressure distribution on the blade of the first impeller under $Q_{\text {des. }}$.

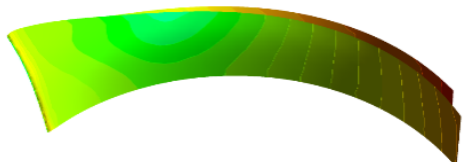

Scheme 1

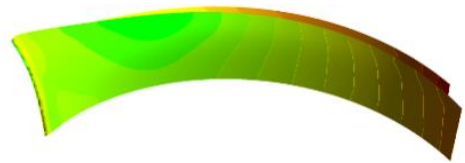

Scheme 2

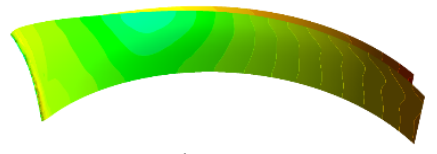

Scheme 3

Figure 10: Static pressure distribution on the blade of the first impeller under $1.4 Q_{\text {des }}$.

Under the $Q_{\text {des }}$ flow rate, the low-pressure region still existed on the blade back face in Scheme 2, whereas the low-pressure regions on the blade back face in Schemes 1 and 3 weakened greatly. In these regions, the pressure gradient of the low-pressure core region in Scheme 1 was not different from that at other positions of the flow field. However, the low- 
pressure core region in Scheme 3 mainly appeared at the first half segment and the last half part of the back face of the blade inlet rim, and a core region fault with a small pressure gradient appeared in the middle region. These occurrences manifested that the constant inlet angle of Scheme 3 could satisfy the distribution of the incoming liquid flow at the centre of the blade inlet rim, but its two sides did not match the angle of the incoming flow to a certain degree. Under the $1.4 Q_{\text {des }}$ flow rate, the discharge capacity of the impeller channel was completely released because the discharge quantity was extremely large, thus greatly inhibiting the flow separation phenomenon caused by the non-uniform speed distribution in the channel. Nevertheless, the high-speed region was formed because the small inlet angle was smaller than the incoming angle that remained in the first half part of blade back face in Scheme 3. In addition, the region's scope was larger than those in Schemes 1 and 2. This lowpressure region continuously diffused in the channel as the flow rate increased, consequently increasing the hydraulic loss and hydraulic efficiency in Scheme 3.

Fig. 11 shows the pressure distribution point clouds on the centre line of the flow channel on the blade surface in the three groups of models. The dispersity of the point cloud distribution represents the pressure difference between the impeller blades at the same position. The figure shows that at the same position of different blades, their static pressure distributions were not identical, indicating that the single-channel numerical calculation method resulted in a certain deviation from the hypothesis that the flow field distributions in each flow channel are the same. From the static pressure distributions on the blade surface in the models under different flow rates the pressure point cloud distribution under the $0.6 Q_{\text {des }}$ flow rate was the most concentrated in Scheme 3. The pressure difference between impeller blades at the same position was the minimum. At the front end of the impeller blade, the minimum pressure at the inlet of the back face was the maximum value among the three groups of models. This result indicated that under the $0.6 Q_{\text {des }}$ flow rate, the blade inlet angle in Scheme 3 could not only prevent the formation of a low-pressure region on the back face at the inlet rim but also contribute to a stable flow field distribution in the last half segment of the impeller flow channel.
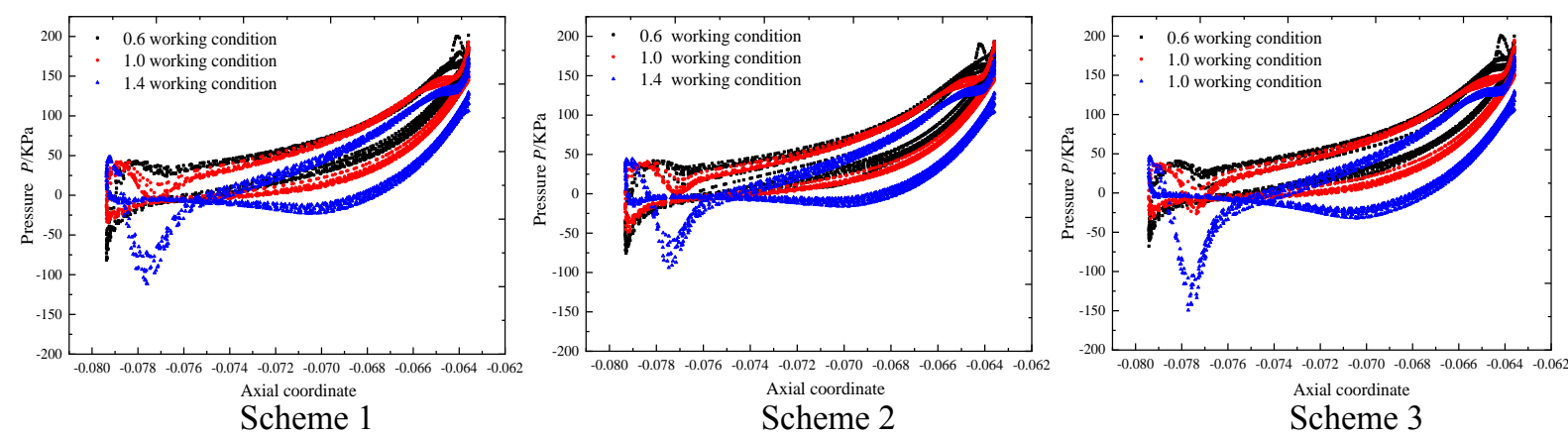

Figure 11: Pressure distribution point cloud with blade surface on the middle line of the flow channel in the three sets of model schemes.

Under the $Q_{\text {des }}$ flow rate, the pressure point cloud distributions in the groups of models indicate a pressure drop at the inlet of the back face and the first half segment of the working face in all models. In these regions, the pressure drop reached the maximum value at the inlet of back face in Scheme 2. In Scheme 3, the pressure drop reached the maximum value in the first half segment of the working face of the blade, and the extreme pressure value at this position was lower than pressure value on the back face under the same axis coordinates. As a result, the turbulent flows of the medium were easily caused, and the stall vortices were formed. The turbulent flow on the working face could easily be diffused in the impeller flow channel; thus, the internal flow field distribution was the most unstable under the rated flow rate in the three groups of models. Under the $1.4 Q_{\text {des }}$ flow rate, the pressure drop in the first half segment of the back face of the blade was evident in all of the models, but the blade inlet 
angle had the highest matching ratio with the incoming flow angle of the liquid medium in Scheme 3. Thus, the pressure drop reached the maximum value. The pressure drop reached the minimum value at the first half segment, and a dense point cloud distribution was observed in the middle segment of the flow channel due to the large inlet angle in Scheme 2. This result indicated that the flow field distribution of Scheme 2 was under a steady state.

\subsection{Experimental analysis of pump performance}

On the basis of the numerical prediction results, a performance test was conducted on the 200QJ50 submersible well pump. Scheme 1 was selected for the prototype manufacturing and performance testing in consideration of the strict performance requirements of the cooperative enterprise for submersible well pumps under a large flow rate.

In the test process of the submersible well pump, a six-stage structure was used for the pump to match the existing motor of specific power. Therefore, the prediction results obtained from the numerical calculation were converted into a six-stage performance. Fig. 12 displays the comparison of the numerical prediction and experimental measurement results of the performance of the 200QJ50 submersible well pump.

The predicted and test values of the head of the 200QJ50 submersible well pump at rated flow rates were identical, with an error of approximately $1 \%$. However, the prediction of shaft power through numerical calculation had a certain error, and the predicted shaft power was lower than the test shaft power by $2.6 \%$. Consequently, under a small deviation in the predicted head value, the prediction efficiency was higher than the test efficiency by $2.3 \%$ because the axial length of the pump increased with the stage number. Thus, the mechanical losses, such as the sealing and filler losses, result in a certain structural deviation in numerical prediction.

The test indicated that the single-stage head of the 200QJ50 submersible well pump was higher than the design requirement, and its efficiency exceeded the national standard requirement.

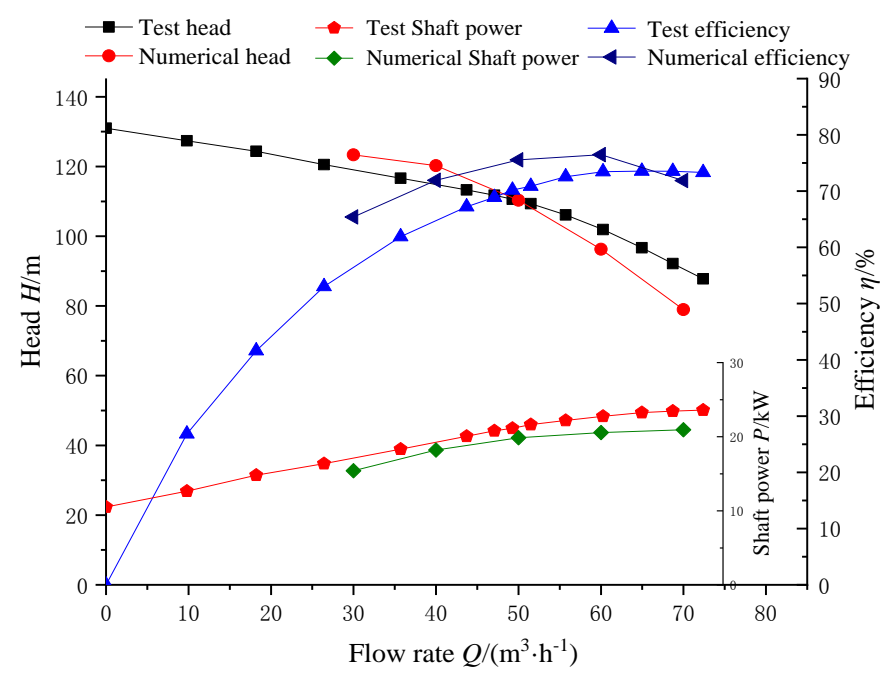

Figure 12: Comparisons between the test and numerical results of the 200QJ50 submersible well pump.

\section{CONCLUSION}

Three different inlet angle schemes were designed to improve the hydraulic design of submersible well pumps and explore the influence mechanism of the change in impeller inlet angle on the performance of submersible well pumps. On the basis of the numerical simulation results, the internal flow characteristics of the three schemes under different working conditions were analysed, and the optimal scheme was verified through experimental comparison. The following conclusions were drawn: 
1) The matching ratio between the blade inlet and incoming flow angles of a fluid is an important factor that influences the performance of submersible well pumps. The design process should emphasize on enlarging the inlet angle but should optimize the parameters instead by following the flow field law.

2) The discharge capacity is consistent with the physical truth under rated flow rates; thus, the improvement of the flow field by the inlet angle is not evident; however, improvement is observable under partial working conditions. Therefore, enlarging the inlet angle can weaken the low-pressure vortex at the inlet and reduce the turbulence of the flow field.

3) In comparison with the hydraulic performance of the pump under the constant inlet angle scheme, that under Scheme 1 (i.e., uniform change of the inlet angle from the front cover plate to the rear cover plate) considerably improved. This result provides a reference for the further development of a follow-up hydraulic design of submersible well pumps.

Numerical simulation was combined with experimental research in this study. Furthermore, a calculation model for the hydraulic analysis of submersible well pumps was proposed. Blade inlet angle, which is an important factor that influences the efficiency of impeller design, was also investigated. This study could guide the optimization of the hydraulic design of submersible well pumps. Only three different schemes were designed in this study; therefore, an optimization analysis method must be considered in the follow-up optimization design of submersible well pumps.

\section{ACKNOWLEDGEMENT}

The study was supported by the Changzhou Sci\&Tech Program (No. CJ20190048) and the Jiangsu Province Graduate Practice Innovation Project (No. SJCX18_0743).

\section{REFERENCES}

[1] Bianchini, A.; Rossi, J.; Antipodi, L. (2018). A procedure for condition-based maintenance and diagnostics of submersible well pumps through vibration monitoring, International Journal of System Assurance Engineering and Management, Vol. 9, No. 5, 999-1013, doi:10.1007/s13198$\underline{018-0711-3}$

[2] Sardjono, P.; Saputra, M. N. W. (2016). Optimal bottomhole pressure control on oil well production using PID-linear hybrid control on electric submersible pump, $20168^{\text {th }}$ International Conference on Information Technology and Electrical Engineering, 6 pages

[3] Shankar, V. K. A.; Umashankar, S.; Paramasivam, S.; Hanigovszki, N. (2016). A comprehensive review on energy efficiency enhancement initiatives in centrifugal pumping system, Applied Energy, Vol. 181, 495-513, doi:10.1016/j.apenergy.2016.08.070

[4] Farid Ayad, A.; Abdalla, H. M.; El-Azm Aly, A. A. (2015). Effect of semi-open impeller side clearance on the centrifugal pump performance using CFD, Aerospace Science and Technology, Vol. 47, 247-255, doi:10.1016/j.ast.2015.09.033

[5] Guo, X.; Zhu, Z.; Cui, B.; Shi, G. (2016). Effects of the number of inducer blades on the anticavitation characteristics and external performance of a centrifugal pump, Journal of Mechanical Science and Technology, Vol. 30, No. 7, 3173-3181, doi:10.1007/s12206-016-0510-1

[6] Wang, C.; Chen, X.; Qiu, N.; Zhu, Y.; Shi, W. (2018). Numerical and experimental study on the pressure fluctuation, vibration, and noise of multistage pump with radial diffuser, Journal of the Brazilian Society of Mechanical Sciences and Engineering, Vol. 40, No. 10, Paper 481, doi:10.1007/s40430-018-1355-6

[7] Zhou, F.-M.; Wang, X.-F. (2016). Effects of staggered blades on the hydraulic characteristics of a 1400-MW canned nuclear coolant pump, Advances in Mechanical Engineering, Vol. 8, No. 8, 21 pages, doi: $10.1177 / 1687814016657944$

[8] Khoeini, D.; Shirani, E. (2018). Enhancement of a centrifugal pump performance by simultaneous use of splitter blades and angular impeller diffuser, International Journal of Fluid Machinery and Systems, Vol. 11, No. 2, 191-204, doi:10.5293/IJFMS.2018.11.2.191 
[9] Bacharoudis, E. C.; Filios, A. E.; Mentzos, M. D.; Margaris, D. P. (2008). Parametric study of a centrifugal pump impeller by varying the outlet blade angle, The Open Mechanical Engineering Journal, Vol. 2, 75-83, doi:10.2174/1874155X00802010075

[10] Nishi, Y.; Fukutomi, J. (2015). Component analysis of unsteady hydrodynamic force of closedtype centrifugal pump with single blades of different blade outlet angles, International Journal of Rotating Machinery, Vol. 2015, Paper 419736, 17 pages, doi:10.1155/2015/419736

[11] Lee, S. H.; Lee, D. R. (2016). Flow analysis of the impeller with different inlet angles in the centrifugal pump, Journal of Computational Fluids Engineering, Vol. 21, No. 1, 58-63, doi:10.6112/kscfe.2016.21.1.058

[12] Rakibuzzaman, M.; Kim, K.; Kim, H.-H.; Suh, S.-H. (2017). Energy saving rates for a multistage centrifugal pump with variable speed drive, Journal of Power Technologies, Vol. 97, No. 2, 163 168

[13] Cheng, X.; Lü, B.; Zhang, X.; Wei, Y.; Zhang, S.; Wang, P. (2018). Influence of outlet edge position of guide vane on performance of well submersible pump, Transactions of the Chinese Society of Agricultural Engineering, Vol. 34, No. 10, 68-75, doi:10.11975/j.issn.1002-6819.2018.10.008

[14] Shi, W.; Zhou, L.; Lu, W.; Xu, L.; Li, W. (2013). Numerical simulation and experimental study of different stages deep-well centrifugal pump, Journal of Computational \& Theoretical Nanoscience, Vol. 10, No. 12, 2897-2901, doi:10.1166/jctn.2013.3297

[15] Wang, H.; Shi, W.; Yang, Y.; Zhou, L.; Lu, W. (2018). Matching number of blades between impeller and diffuser blade effect on performance of well submersible centrifugal pump, Transactions of the Chinese Society for Agricultural Machinery, Vol. 49, No. 7, 103-111, doi:10.6041/j.issn.1000-1298.2018.07.013

[16] Stel, H.; Sirino, T.; Ponce, F. J.; Chiva, S.; Morales, R. E. M. (2015). Numerical investigation of the flow in a multistage electric submersible pump, Journal of Petroleum Science and Engineering, Vol. 136, 41-54, doi:10.1016/j.petrol.2015.10.038

[17] El-Gazzar, D. M. S.; Hawash, S. A.-A. F. (2018). Optimizing centrifugal pump performance by different blade configuration patterns, American Journal of Mechanical and Industrial Engineering, Vol. 3, No. 1, 1-14, doi:10.11648/j.ajmie.20180301.11

[18] Gölcü, M.; Pancar, Y.; Sekmen, Y. (2006). Energy saving in a deep well pump with splitter blade, Energy Conversion and Management, Vol. 47, No. 5, 638-651, doi:10.1016/ j.enconman.2005.05.001

[19] Wei, Q.; Sun, X. (2017).Performance influence in submersible pump with different diffuser inlet widths, Advances in Mechanical Engineering, Vol. 9, No. 1, 8 pages, doi:10.1177/ 1687814016683354

[20] Ajay, A. J.; Stephen, S. E. A.; Smart, D. S. R. (2017). Shape optimization of submersible pump impeller design, International Conference on Recent Advances in Aerospace Engineering, 6 pages

[21] Lu, Z.; He, X.; Wang, C. (2018). Influencing factors of self-priming time of multistage selfpriming centrifugal pump, DYNA, Vol. 93, No. 6, 630-635, doi:10.6036/8930

[22] Zhou, L.; Shi, W.; Wu, S. (2013). Performance optimization in a centrifugal pump impeller by orthogonal experiment and numerical simulation, Advances in Mechanical Engineering, Vol. 5, Paper 385809, 7 pages, doi:10.1155/2013/385809

[23] Kim, J.-H.; Lee, H.-C.; Kim, J.-H.; Kim, S.; Yoon, J.-Y.; Choi, Y.-S. (2015). Design techniques to improve the performance of a centrifugal pump using CFD, Journal of Mechanical Science and Technology, Vol. 29, No. 1, 215-225, doi:10.1007/s12206-014-1228-6

[24] Li, W.; Zhou, L.; Shi, W.; Ji, L.; Yang, Y.; Zhao, X. (2017). PIV experiment of the unsteady flow field in mixed-flow pump under part loading condition, Experimental Thermal and Fluid Science, Vol. 83, 191-199, doi:10.1016/j.expthermflusci.2017.01.009

[25] Capurso, T.; Bergamini, L.; Torresi, M. (2019). Design and CFD performance analysis of a novel impeller for double suction centrifugal pumps, Nuclear Engineering and Design, Vol. 341, 155166, doi:10.1016/j.nucengdes.2018.11.002

[26] Jiang, X.; Wang, C.; Wu, J.; Shi, W.; Liu, S.; Yang, Y. (2017). Effect of diffuser inlet width on cantilever multistage centrifugal pump, DYNA, Vol. 92, No. 1, 63-70, doi:10.6036/8153

[27] Namazizadeh, M.; Gevari, M. T.; Mojaddam, M.; Vajdi, M. (2020). Optimization of the splitter blade configuration and geometry of a centrifugal pump impeller using design of experiment, Journal of Applied Fluid Mechanics, Vol. 13, No. 1, 89-101, doi:10.29252/jafm.13.01.29856 